\title{
Adrenal cortical tumor with rare presentation of psychosis
}

\begin{abstract}
Adrenal cortical tumors are rare tumors and the common presenting symptoms are related with excessive glucocorticoids secretions leading to cushinoid features and systemic manifestations of the excess steroids/hormonal secretions. We are presenting a case of Adrenal cortical tumor presenting as primarily psychosis hence Adrenal cortical tumor should always be considered in the differential diagnosis of evaluation of psychosis.
\end{abstract}

Keywords: adrenal cortical tumor, cushinoid features, glucocorticoids, psychosis
Volume 3 Issue 4 - 2016

\author{
Asadullah Nawazani,' Mayank G Vats, ${ }^{2}$ \\ Faiz ur rehman farooqui, ${ }^{3}$ Hina Zia Mirza, ${ }^{3}$ \\ Mohammed Jamshed Khan, ${ }^{3}$ Samreen \\ Nawazani ${ }^{4}$ \\ 'Departmentof Medicine, Dubai hospital, UAE \\ ${ }^{2}$ Department of Respiratory Medicine and Sleep Medicine, UAE \\ ${ }^{3}$ Department of Medicine, UAE \\ ${ }^{4}$ The Elite English School, UAE
}

Correspondence: Mayank G Vats, Senior Specialist, Department of Respiratory Medicine and Sleep Medicine, Rashid Hospital, Dubai, UAE,Tel +97I-558825307, Email drmayankvats@gmail.com

Received: May 29, 2016 | Published: June 13, 2016

\section{Introduction}

Adrenal cortical hormone producing tumors are rare but commonly presents as plethora of manifestations of glucocorticoids excess secreted by the tumor. However they rarely can present as psychiatric manifestations alone. We are presenting a case of adrenal cortical tumor presenting as Frank psychosis and hence adrenal cortical tumors should be considered in differential diagnosis of such patients.

\section{Case presentation}

We are presenting a case report of 64years old lady, who doesn't have any significant past medical or surgical history. Her symptoms started $1 \frac{1}{2}$ month prior to admission, when her family noticed a change in her personality. She became aggressive, agitated, loose her temperament easily and spent most of time in isolation. Along with these personality changes she complains of excessive sweating, drenching of clothes while sleeping at night. After few weeks of symptoms family decided to seek medical checkup in some private hospital. Where she was diagnosed diabetes Mellitus and hypertension, she was also told to have abdominal masses and advised for further investigations.

She was brought by family in our ER with psychotic features, visual hallucinations, agitation, biting peoples around her and violent movements of limbs. She was made calm with small dose of haloperidol. On examination, vitals were stable including BP 157/98mmhg, HR 119/minute. Temperature 36.7degree centigrade, SpO2 99\% on room air. GCS-15/15, she was agitated with facial acne (Figure 1) along with features of hirsutism and central obesity.

Per Abdomen examination revealed soft, not tender abdomen, light purple striae on flanks bilaterally (Figure 2) and a mass palpable in left lumber region along with palpable liver. Chest auscultation revealed normal vesicular breath sounds, CVS: normal heart sounds, no murmur. CNS: No focal neurologic deficit, no neck stiffness.

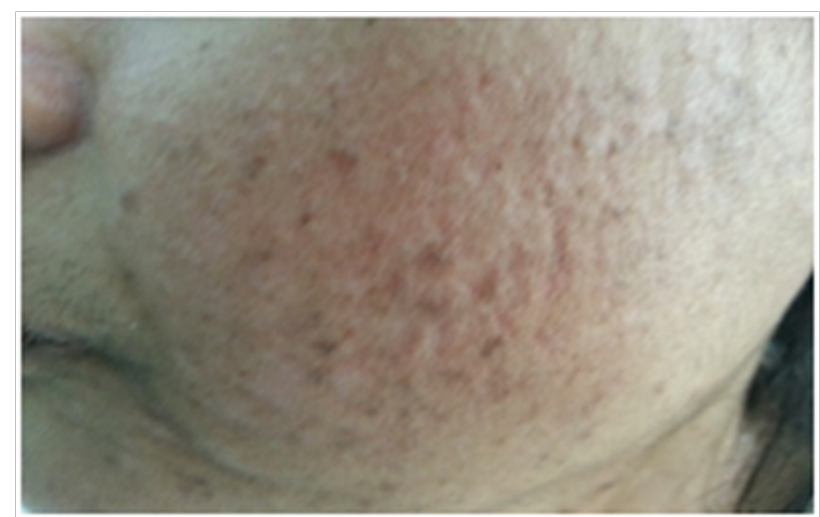

Figure I Shows acniform lesions on face.

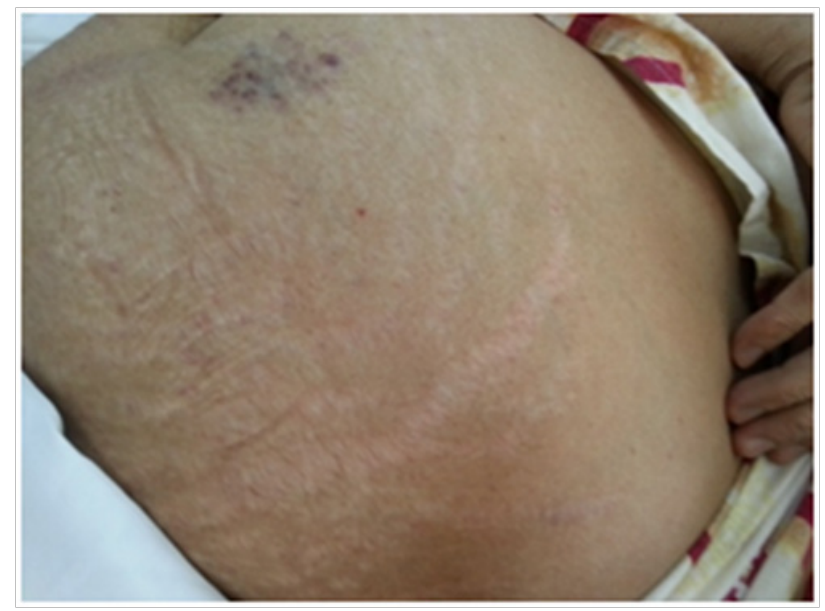

Figure 2 Showslight purple striae on flanks bilaterally. 
CT scan abdomen revealed Peripheral-sub capsular- large right hepatic heterogeneous well-defined lesion is seen measuring $13 \times 9 \mathrm{~cm}$ showing peripheral wall enhancement \& heterogeneous internal enhancement noted in arterial phase and persist in venous with internal vessels seen (Figure 3). Complete wash out in delayed image noted. Other small satellite hypo attenuated lesion is seen medially measuring $1.4 \times 0.9 \mathrm{~cm}$ showing heterogeneous pattern. Other large lobulated heterogeneous left suprarenal lesion is seen measuring $12 \times 7 \mathrm{~cm}$ showing cystic/necrotic anterolateral portion and heterogeneous enhancement (Figure 4). This mass is seen separated from the left kidney \& indenting the displacing the pancreatic tail anteriorly. The left adrenal gland is not seen. Routine blood tests were unremarkable.

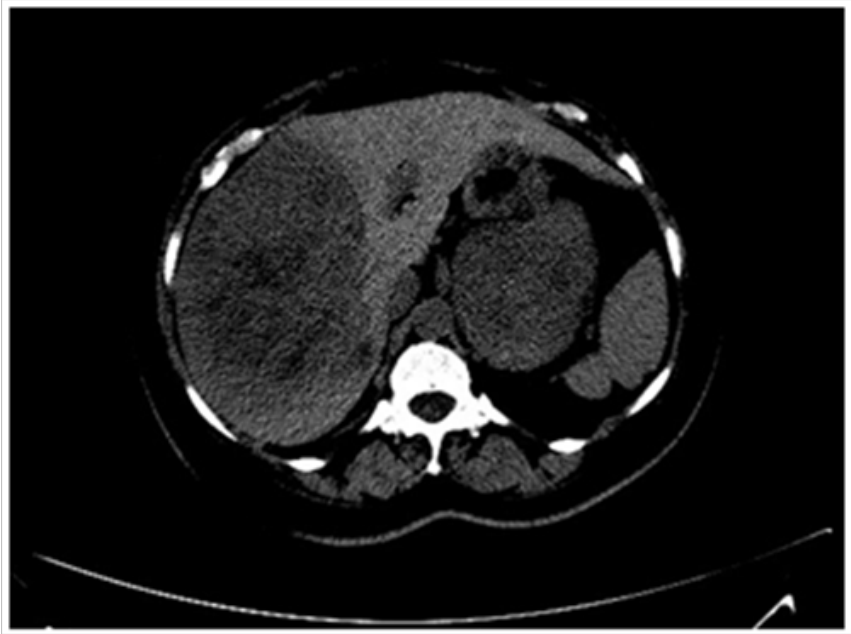

Figure 3 CT Scan Abdomen: Reported a liver mass of about $13 \times 9 \mathrm{~cm}$ size.

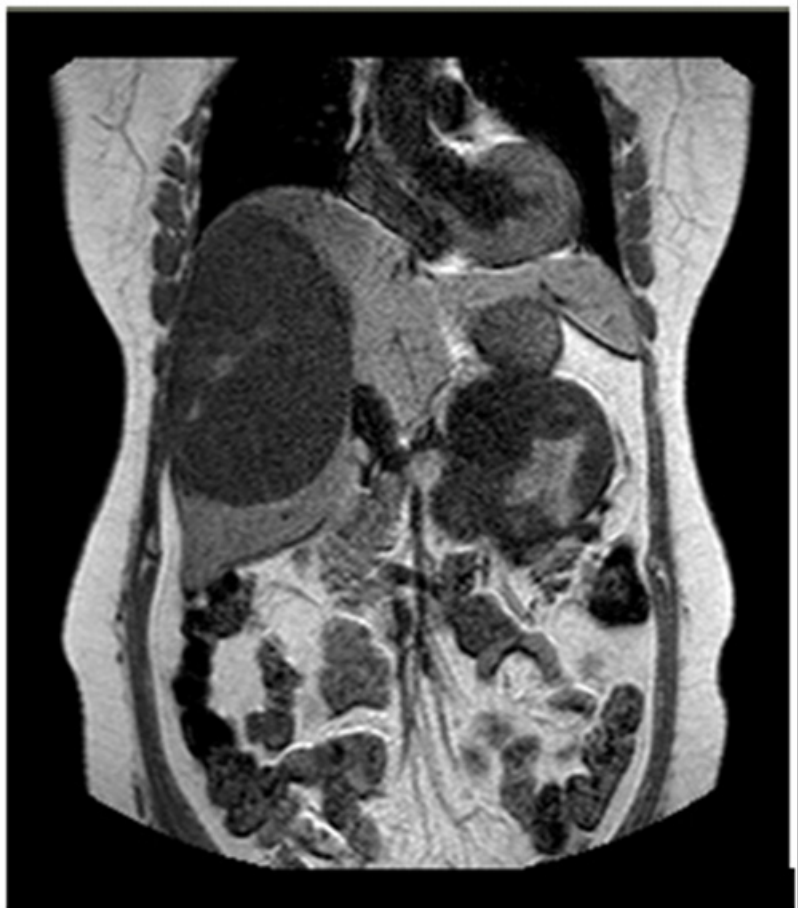

Figure 4 MRI Abdomen: Reported a liver lesion of $12.4 \times 9 \mathrm{~cm}$ \& adrenal lesion $12 \times 8.7 \mathrm{~cm}$ size.

\section{Hormone profile (range) shows}

Cortisol AM 1350nmol/L ( range 138- 690) , Cortisol Dynamic $1486 \mathrm{nmol} / \mathrm{L}$, Dexamethasone suppression test: $1430 \mathrm{nmol} / \mathrm{L}$, Urinary cortisol 24H : 635ug/24H (range 20-90), ACTH am $<5$ (range $<46$ ), Testosterone: $18 \mathrm{nmol} / \mathrm{L}$, (Menopause 0.28-1.2), Free Androgen Index 180 (range 0.8-6 ), SHBG 10nmol/L (range 2085), DHEA (dehydroepiandrosterone) Sulphate: $>1200$ (range 25-182),Androstenedione: $>30$ (range1.7-16.2) , 17-OH progesterone $-9.8 \mathrm{mmol} / 1$ ( menopause range 0.7-4.1), Prolactin 299mIU/L (range 102- 496), Renin erect 60uIU/ml (range 2.9-30), Aldosterone upright : 110pmol /1 ( adult range 97-833), Chromogranin A : 145+ $\mu$ g (range $<85)$, Serum Metanephrine $<50 \mathrm{ng} / \mathrm{L}(<90)$, Serum Nor-metanephrine $<50 \mathrm{ng} / \mathrm{L}(<129)$, Adrenaline $<20 \mathrm{ng} / \mathrm{L}$ (upto 84$)$, Noradrenaline 170ng/L (upto 420), Dopamine $<20$ (upto 85 ).

CT scan guided liver biopsy was done and reported as Metastatic carcinoma, morphologically and immunohistochemically goes with adrenal gland cortical origin (adrenal cortical carcinoma) (Figure 5).

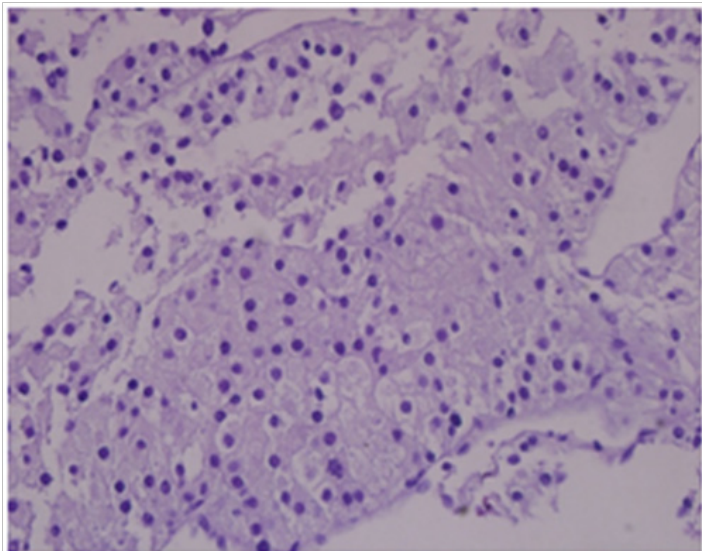

Figure 5 H \& E Staining: Shows malignant cells.

Immuno-histochemical studies of the tumor was as follows: Melan A: Positive (Figure 6), Alpha inhibin: Positive (Figure 7), Calretinin: Negative, Chromogranin: Negative, Renal cell carcinoma (RCC): Negative, Hep Par 1: Negative hence confirming the diagnosis of adrenal cortical carcinoma.

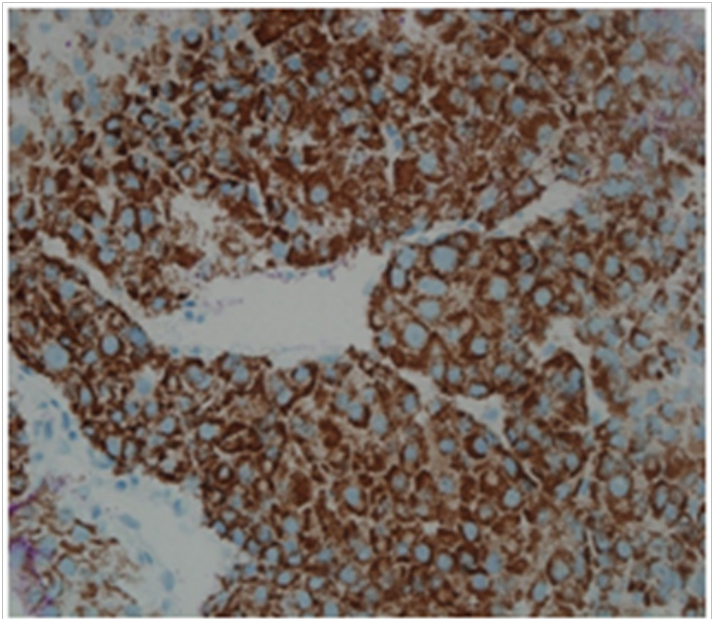

Figure 6 Melan A immunostaining shows diffuse positive staining in the cytoplasm of the tumor cellsfavoring adrenal tumor. 


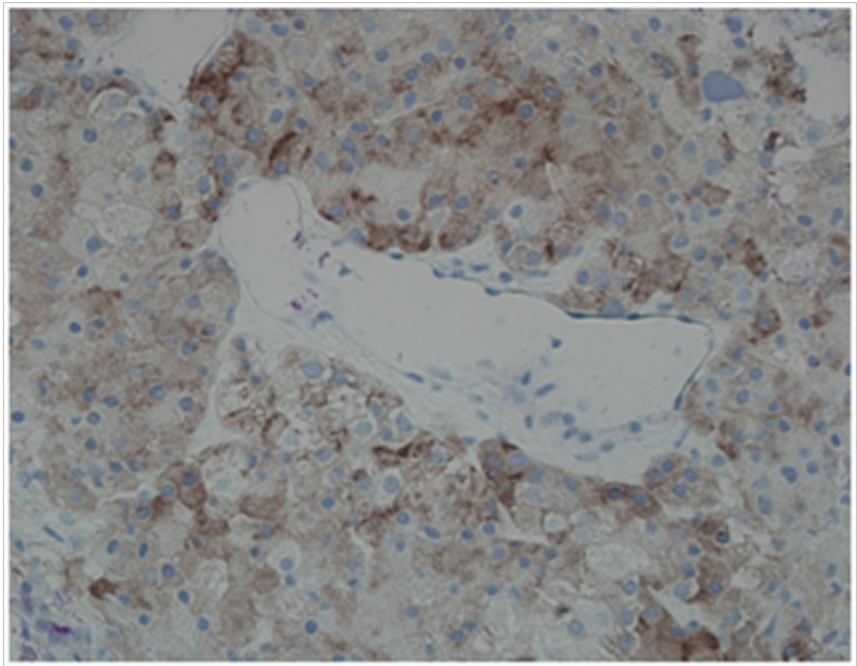

Figure 7 Inhibin positive immunostaining favors tumor from adrenal origin.

Hospital course: Patient was ambulatory and hemodynamicaly stable. She continued to have brief episodes of psychosis which were controlled with haloparidol. Her cortisol levels were controlled by ketoconazole, with some improvement in her mood and psychosis. Subsequently, she was referred to oncology department for further management.

\section{Discussion}

Adrenal glands are located deep in the abdominal cavity; because of their location it is common to diagnose this tumor late with frequent metastasis to other organs as in our case.

Adrenal cortical tumor is extremely rare cancer. It has annual incidence of 2 per million of population. ${ }^{1}$ This tumor occurs in late adulthood, $5^{\text {th }}$ decade of life $^{2}$ and rarely in children $<5 y$ years, ${ }^{3}$ the median age in adult is 46 years. When occurs in children it has genetic predisposition.

About $30 \%$ of adrenal tumors are malignant. They commonly metastasize to liver, lungs, and peritoneum and less commonly to bones and veins. Commonly, produced hormones are cortisol, androgens (testosterone, dehydroepiandosterone), as in this case however tumor can produce and cachcolamines though less commonly. Increased serum levels of Chromogranin A are found in different neuroendocrine tumors (Pheochromocytoma, Carcinoid, C-cell-carcinoma, and Isletcell-carcinoma, hypophysial tumors, small cell-bronchial-carcinoma and patients with renal insufficiency.

It is not surprising for these functioning tumors to mimic other disease conditions. About $60 \%$ of adrenal cortical tumors are symptomatic because of excessive hormone secretion. The common symptoms are palpitations, nervousness, headache, anxiety or panic attacks, abdominal pain or heaviness, unexplained weight gain or weight loss, weakness, abdominal stretch marks, excessive hair growth, unusual acne, change in libido, excessive perspiration and dyspnea. ${ }^{4}$ Of these symptoms, this lady has perspiration at night and acniform eruptions on her face; she denied symptoms of mass effect in her abdomen.

Many patients with adrenal tumors secrete glucocorticoid and develop cushinoid features., ${ }^{3,5}$ However, in our case patient had hypersecretion of both glucocorticoids and androgens, so called mixed pattern, reported in $35 \%$ of patients. ${ }^{6}$

She developed frank psychosis along with cushinoid features that happens in only $25 \%$ of cases. Though it is well known that excess glucocorticoids cause psychosis however to our knowledge it is not reported in adrenal cortical carcinoma before.

Mood changes are common in secreting tumors of adrenals. ${ }^{7}$ In one study major depressive syndrome was seen in $50 \%-70 \%$ of the cases. ${ }^{8}$ Other associated features include anxiety in $12 \%-79 \%$ of the cases, ${ }^{9}$ and hypomania in $3 \% .{ }^{10}$ Less common are features of psychosis and mania. ${ }^{11,12}$

Patient also present with new onset of high blood pressure and diabetes mellitus most likely because of excessive cortisol secretion.

She was given cortisol secretion blocker medications, however her symptoms decreased marginally, literature reported that even well treated patients can persistently have psychiatric symptoms for long time. Overall survival rate in these tumors is dismal. However, 10year survival has been reported, but recurrence is very common.

Only effective treatment possibly curative is surgery, ${ }^{13}$ chemotherapy, DDD and radiotherapy are effective in few numbers of patients.

Generally adrenal cortical tumor when produces hormones has unfavorable prognosis and have a grave prognosis after metastasizes to distant organs.

\section{Conclusion}

Adrenal cortical tumors most be suspected in a patient presenting with psychosis and features of cushinoid syndrome and adrenal cortical tumor should be considered as differential diagnosis for evaluation of the new onset psychosis of no cause could be find while evaluating a case of Psychosis.

\section{Acknowledgements}

Dr Asadullah and Dr Mayank prepared the case presentation, manuscript writing. Dr Faiz, Dr Hina \& dr Samreen did the literature and manuscript review \& formatting.

\section{Conflict of interest}

The author declares no conflict of interest.

\section{References}

1. Robin PB, Alan PBD. Adrenal cortical carcinoma. Current Treatment Options in Oncology. 2001;2(4):355-364.

2. Wooten MD, King DK. Adrenal cortical carcinoma. Epidemiology and treatment with mitotane and a review of the literature. Cancer. 1993;72(11):3145-3155.

3. Icard P, Chapuis Y, Andreassian B, et al. Adrenal cortical carcinoma in surgically treated patients: a retrospective study on 156 cases by the French association of endocrine surgery. Surgery. 192;112(6):972-979.

4. Pommier RF, Brennan MF. An eleven year experience with adrenocortical carcinoma. Surgery. 1992;112(6):963-970.

5. Proye CAG, Pattou FN. Adrenocortical carcinoma: nonfunctioning and functioning. Textbook of Endocrine Surgery. Philadelphia, Saunders; 1997. p. $490-496$ 
6. Dackiw AP, Lee JE, Gagel RF, et al. Adrenal cortical carcinoma. World J Surg. 2001;25(7):914-926.

7. Arnaldi G, Angeli A, Atkinson B, et al. Diagnosis and complications of Cushing's syndrome:a consensus statement. J Clin Endocrinol Metab. 2003;88(12):5593-5602.

8. Sonino N, Fava GA. Psychosomatic aspects of Cushing's disease. Psychother Psychosom. 1998;67(3):140-146.

9. Loosen PT, Chambliss B, DeBold CR, et al. Psychiatric phenomenology in Cushing's disease. Pharmacopsychiatry. 1992;25(4):192-198.

10. Kelly WF, Kelly MJ, Faragher B. A prospective study of psychiatric and psychological aspects of Cushing's syndrome. Clin Endocrinol. 1996;45(6):715-720.
11. Katho RG, Delahunt JW, Hannah L. Transition from bipolar affective disorder to intermittent Cushings's syndrome: case report. J Clin Psychiatry. 1985;46(5):194-196.

12. Hirsh D, Orr G, Kantarovich V, et al. Cushing's syndrome presenting as a schizophrenia-like psychotic state. Isr J Psychiatry Relat Sci. 2000;37(1):46-50

13. Hajjar RA, Hickey RC, Samaan NA. Adrenal cortical carcinoma: A study of 32 patients. Cancer. 1975;35(2):549-554. 